\section{IDDF2021-ABS-0055 ANALYSIS OF EXPOSURE-OUTCOMES OF USTEKINUMAB IN PATIENTS WITH REFRACTORY CROHN'S DISEASE}

Jiayin Yao*, Min Zhang, Tao Liu, Min Zhi. Department of Gastroenterology, The Sixth Affiliated Hospital of Sun Yat-Sen University, China

\subsection{6/gutjnl-2021-IDDF.121}

Background Ustekinumab (UST), a newly-used biologics targeting p40 subunit of IL12 and IL23 in China, exerts confirmed therapeutic effect in induction and maintenance therapy of refractory Crohn's disease (CD). Therapeutic drug monitoring based on trough and antibody concentration is of core importance when treating patients who lose response to UST. We aimed to analyze UST exposure-response relationship in CD treatment.

Methods We retrospectively enrolled CD patients with UST administration between March 1, 2020 and May 31, 2021 at IBD center of the Sun Yat-Sun Affiliated Sixth Hospital. Baseline characteristic information, biomarker examination, clinical outcomes determined by Crohn's disease activity index (CDAI), and endoscopic outcomes evaluated by a simple endoscopic score for Crohn's disease (SES-CD)at week 16/20 week were collected. The optimal UST trough concentration was identified by receiver-operating characteristic curve (ROC). Results Nineteen eligible patients were finally included, with mean ages as 29.19.1 and mean disease durations as 5.54.7. At study initiation, $89.5 \%$ patients had been exposure to prior biologics, $42.1 \%$ had previous CD-related surgeries, and $52.6 \%$ had a perianal disease. At week 16/20 after UST initiation, clinical response, clinical remission, endoscopic response, and endoscopic remission were $89.5 \%, 84.2 \%, 42.2 \%$, and $73.7 \%$, respectively. The cut-off optimal trough concentration of UST was $1.12 \mu \mathrm{g} / \mathrm{mL}$ evaluated by ROC with AUC as 0.78 , sensitivity as $87.5 \%$, and specificity as $72.7 \%$ (IDDF2021ABS-0055 Figure 1. Receiver-operating curve analysis for endoscopic remission based on UST trough concentration with optimal UST trough level cut-offs of $1.12 \mu \mathrm{g} / \mathrm{mL}$ ). Patients with a UST trough concentration $>1.12 \mu \mathrm{g} / \mathrm{mL}$ had a significantly higher rate of endoscopic remission than those without

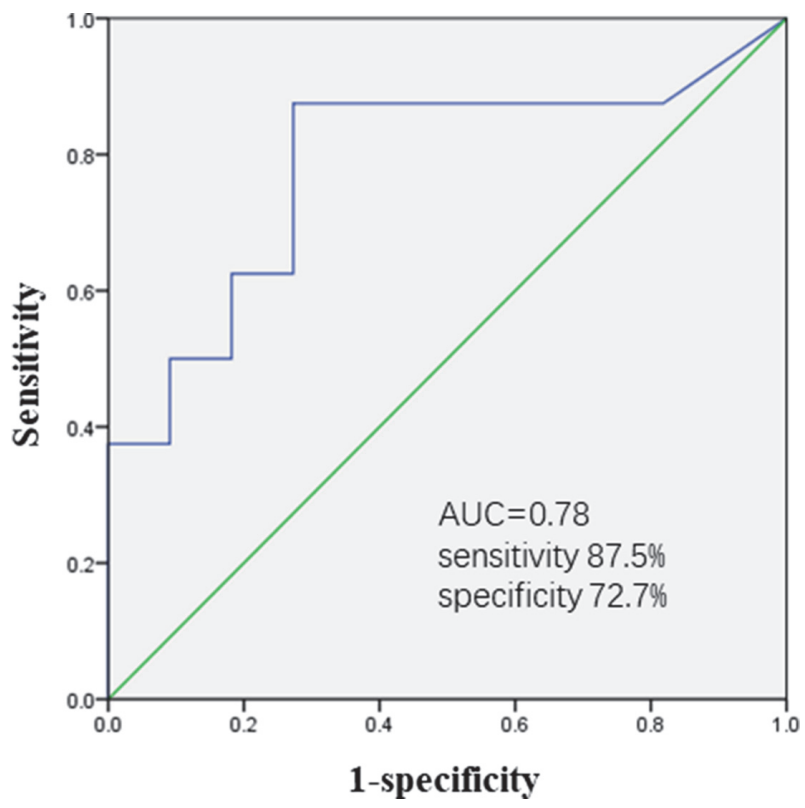

Abstract IDDF2021-ABS-0055 Figure 1

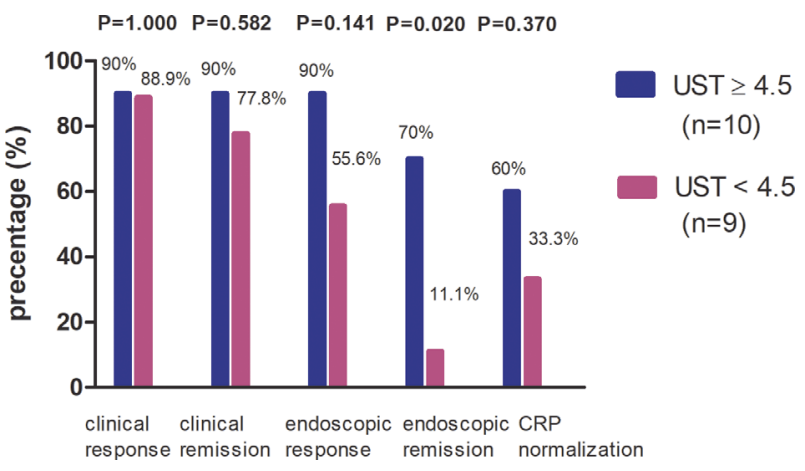

Abstract IDDF2021-ABS-0055 Figure 2

(70.0\% vs. $11.1 \%, P=0.02$ ) (IDDF2021-ABS-0055 Figure 2. Clinical and endoscopic outcomes based on UST trough concentration).

Conclusions UST is an effective therapy weapon dealing with refractory CD. Trough concentration of UST above $1.12 \mu \mathrm{g} / \mathrm{ml}$ was associated with endoscopic remission at week 16/20 after UST initiation.

\section{IDDF2021-ABS-0057 OUTCOME OF PER-ORAL ENDOSCOPIC MYOTOMY IN CHILDREN AND ADOLESCENTS WITH ACHALASIA CARDIA: A SYSTEMATIC REVIEW AND META- ANALYSIS}

Zaheer Nabi*, Rupjyoti Talukdar, Manohar Reddy, Rakesh Kalapala, Rama Kotla, Rajesh Goud, Santosh Darisetty. Asian Institute of Gastroenterology, India

\subsection{6/gutjnl-2021-IDDF.122}

Background Per-oral endoscopic myotomy (POEM) is an established treatment modality in adult patients with achalasia cardia. Emerging data suggest that POEM may be effective in pediatric cases as well. In this systematic review and metaanalysis, we aim to analyze the outcomes of POEM in children and adolescents with achalasia cardia.

Methods A literature search was performed in Embase, PubMed, Cochrane database for studies pertaining to POEM in pediatric achalasia between Jan 2010 to March 2013. The primary objective of the study was a clinical success (Eckardt $\leq 3)$. Secondary outcomes included technical success, post-POEM manometry parameters, adverse events and gastroesophageal reflux (GER) after POEM.

Study name
Tang 2015
Chen 2015
Caldaro 2015
Tan 2016
Stavropoulos 2017
Zangen 2017
Korrapati 2018
Nishimoto 2018
Mangiola 2018
Miso 2018
Yamashlta 2018
Nabi 2019
Chone 2019
Wood 2020
Saez 2020
Uiu 2020

\begin{tabular}{ccccc}
\multicolumn{6}{c}{ Statistics for each study } \\
$\begin{array}{c}\text { Event } \\
\text { rate }\end{array}$ & $\begin{array}{c}\text { Lower } \\
\text { lilit }\end{array}$ & $\begin{array}{c}\text { Upper } \\
\text { limit }\end{array}$ & 2 -value & p-value \\
0.900 & 0.326 & 0.994 & 1.474 & 0.140 \\
0.981 & 0.764 & 0.999 & 2.781 & 0.005 \\
0.950 & 0.525 & 0.997 & 2.029 & 0.042 \\
0.962 & 0.597 & 0.998 & 2.232 & 0.026 \\
0.955 & 0.552 & 0.997 & 2.103 & 0.035 \\
0.917 & 0.378 & 0.995 & 1.623 & 0.105 \\
0.969 & 0.650 & 0.998 & 2.390 & 0.017 \\
0.923 & 0.609 & 0.989 & 2.387 & 0.017 \\
0.981 & 0.756 & 0.999 & 2.753 & 0.006 \\
0.977 & 0.723 & 0.999 & 2.629 & 0.009 \\
0.938 & 0.461 & 0.996 & 1.854 & 0.064 \\
0.930 & 0.805 & 0.977 & 4.327 & 0.000 \\
0.946 & 0.886 & 0.976 & 6.843 & 0.000 \\
0.857 & 0.639 & 0.953 & 2.873 & 0.004 \\
0.917 & 0.378 & 0.995 & 1.623 & 0.105 \\
0.956 & 0.898 & 0.981 & 6.717 & 0.000 \\
0.942 & 0.915 & 0.961 & 13.091 & 0.000
\end{tabular}

Abstract IDDF2021-ABS-0057 Figure 1 